\title{
Experimal study of young male drivers' responses to vehicle collision using EMG of lower extremity
}

\author{
Zhenhai Gao ${ }^{\mathrm{a}, \mathrm{b}, \mathrm{c}}$, Chuzhao $\mathrm{Li}^{\mathrm{a}}$, Hongyu Hu${ }^{\mathrm{a},{ }^{*}}$, Hui Zhao ${ }^{\mathrm{b}, \mathrm{c}}$, Chaoyang Chen ${ }^{\mathrm{d}}$ and Huili Yu ${ }^{\mathrm{b}, \mathrm{c}}$ \\ ${ }^{a}$ State Key Laboratory of Automobile Simulation and Control, Jilin University, Changchun 130025, \\ P.R. China \\ ${ }^{b}$ State Key Laboratory of Vehicle NVH and Safety Technology, Changan Automobile Holding Ltd, \\ Chongqing 401120, P.R. China \\ ${ }^{c}$ Changan Automobile Holding Ltd. Automotive Engineering Institute, Chongqing 401 120, P.R. China \\ ${ }^{d}$ Spine Research Laboratory, Department of Biomedical Engineering, Wayne State University, Detroit, \\ MI 48201, USA
}

\begin{abstract}
A driver's response to a front-coming vehicle collision consists of braking reaction time and braking behavior. The purpose was to investigate drivers' responses at different speeds, relative distances, and particularly the behavior on the accelerator at the collision moment. Twelve young men participated in driving simulator tests. Vehicle parameters and electromyograms (EMGs) of the drivers' tibialis anterior muscles were recorded and responses were analyzed. The drivers' braking reaction time windows were divided into pre-motor time, muscle activation time, accelerator release time, and movement time. By comparing the reaction times and collision times, braking behaviors were investigated. It was found that movement times $(r=-0.281)$ decreased with speed. Pre-motor times $(r=0.326)$ and muscle activation times $(r=0.281)$ increased with relative distance. At the collision moment, the probability of the driver's lower extremity being on the accelerator, in the air, and on the brake pedal was $7.4 \%, 18.9 \%$, and $73.7 \%$, respectively. With higher speeds and smaller distances, the lower extremity was more likely to be in the air or even on the accelerator in different muscle activation states. The driver will collide in normal driving postures which muscles are not or not fully activated in very urgent situation.
\end{abstract}

Keywords: Drivers' responses, braking reaction time, braking behavior, collision, electromyogram

\section{Introduction}

Although annual vehicle collision incident rates are decreasing, there were 5,615,000 policereported traffic crashes in 2012 (DOT HS 812 016). Among them, 33,561 people were killed and 2,362,000 injured. Therefore, a better understanding of drivers' reaction and braking kinematic behaviors are vital for biomechanics analysis and vehicle crash prevention.

A driver's response to a collision consists of two parts: braking reaction time and kinematic braking

\footnotetext{
* Address for correspondence: Hongyu Hu, State Key Laboratory of Automobile Simulation and Control, Jilin University, Changchun 130025, P.R. China. Tel.: +86 (0431) 85095090; Fax: +86 (0431) 85682227; E-mail: huhongyu_jlu@126.com.
}

0959-2989/15/\$35.00 @ 2015 - IOS Press and the authors. 
behavior. Braking reaction time represents the driver's ability to react to a collision and the kinematic braking behavior is the operation of the pre-crash posture of the driver's lower extremity reacting under a specific condition. The braking reaction time is one of the important factors when designing the intervention time of active vehicle systems such as Electronic Stability Program (ESP) and Forward Collision Warning system (FCW). Based on a driver's reaction capacity, different protective strategies for occupants should be considered in the design of vehicle safety systems.

When facing a front-coming collision, a driver assumes a protective pre-crash posture, such as bracing and muscle tensing [1,2], or even performs evasive steering maneuvers [3]. These will result in different musculoskeletal characteristics and great influence on the kinematic responses and injuries during and following a collision [4]. Bose analyzed injuries in different postures and discovered that the most severe injuries occurred in out-of-position (OOP) conditions [5]. However, it was uncertain what posture the lower extremity was in when the collision occurred. This uncertainty challenges the trauma biomechanics analysis and vehicle safety system design. Thus, it is necessary to investigate pre-crash postures of drivers' lower extremities.

The drivers' braking reaction times in existing studies were divided into three parts, which included brake reaction times (time between the onset of the brake lights of the target car and the release of the accelerator pedal of the host car), movement times (time between the release of the accelerator pedal and the depression of the brake pedal), and total brake times (the sum of the former two) $[4,6]$. These data were acquired from the vehicle parameters such as accelerator apertures and braking pedal forces. To design vehicle safety systems, it is critical to understand the reaction capacity of the driver [7]. Studying human reaction mechanisms makes it possible to measure drivers' responses more accurately.

In psychology, an electromyogram (EMG) is used to divide braking reaction times into pre-motor time and motor time [8]. Mero and Komi introduced this method into the field of sport physiology to identify pre-motor time and motor time components in sprint start [9]. In recent years, the use of EMG was introduced to the field of accidental injury biomechanics in traffic and sports. Choi, et al. investigated the muscle activation level when drivers were facing unavoidable collisions, and the data were used for human model engineering [10]. Behr, et al. analyzed EMGs to explore drivers' braking postures when facing unexpected emergencies [4]. Combining these two fraction methods of reaction times and measuring vehicle parameters and EMGs of drivers' lower extremities are necessary to study biomechanics in accidental injuries, and for the design of occupant protection systems.

Former studies have shown that a driver's braking reaction time decreases with speed and increases with relative distance $[6,11]$. However, this was based on the traditional division method of reaction times. In this study, the drivers' braking reaction time can be divided into different factors through the use of EMGs. The influences of driving speeds and relative distances on reaction times can be influential in the future design of vehicle safety systems, although more detailed research is still necessary.

Physiological limits exist for human reaction capabilities, and a driver's braking reaction time varies with different driving speeds and relative distances $[6,11]$. This means that a driver cannot respond to a driving situation that exceeds their physiological reaction limit. Usual braking includes two steps: the release of the accelerator and then the application of the brake; this entire reaction is lengthy, and a collision can occur at any time [12]. According to musculoskeletal biomechanics, muscle activation takes time, and must happen before the accelerator is released, so the collision might even occur when the foot is still on the accelerator, depending on the different muscle activation states [13]. Muscle activation of the lower extremity varies at different braking stages, so the driver's braking posture and possible crash injuries are influenced by the braking reaction time in 
a collision. Most studies concerning crash injuries have found that the lower extremity is on the brake pedal in vehicle collisions $[14,15]$. In this research, the drivers' braking reaction times were investigated and muscle activation moments in braking motions at different values of speed and distance parameters were carefully measured.

A driving simulator was used as the testing platform to simulate collisions and to record drivers' responding behaviors in this study. Vehicle parameters and EMGs of the drivers' lower extremities were recorded. The drivers' braking reaction times were measured, detail fractionated, and analyzed. Based on the fraction of braking reaction times and comparisons between collision times and braking reaction times, the braking behaviors of the lower extremities were studied when facing different oncoming collision scenarios.

\section{Methods}

\subsection{Participants}

Twelve male drivers between the ages of 23 and $27(24.7 \pm 1.4)$ participated in this experiment. Each participant had a valid driver's license and several years' driving experience ( $3.9 \pm 0.9$ years). All participants signed informed consent forms before the tests were performed. The study was approved by the First Hospital of Jilin University Ethics Committee.

\subsection{Experimental design and procedure}

The experiments were performed in a driving simulator at the State Key Laboratory of Automobile Simulation and Control, Jilin University, China, as shown in Figure 1. The simulator system included a host computer, a NI real-time target computer with a multifunctional DAQ board (National Instrument, model 8180, Austin, TX), an accelerator pedal, and a brake pedal with a load cell transducer (EVT-14W, Shanghai Yu Ran Sensor Technology Co., Ltd) on it. The driving simulator was mounted on a Stewart platform, in which the yaw, pitch, and roll motion of the vehicle could be performed. The simulator was used to replicate frontal collision scenarios while the drivers' operation parameters and data from the accelerator/brake pedals and steering wheel were recorded. Meanwhile, the simulator was equipped with a realistic control operation system, such as a steering wheel with force feedback, a brake pedal with power assist feel, and a stereo sound system that mimicked driving noise.

According to the annual report of road accidents in China for 2013, two-way roads with two lanes make up $95.1 \%$ of the total miles of roads. Driving in the on-coming lane and overtaking illegally are main causes of road accidents, and frontal collisions are common. Based on the method used by McGehee [1], a driving scenario using a 2 x 30-km-long two-way rural highway was constructed. The host car was driven by the participant in one lane at a designated speed. Opposing-lane traffic

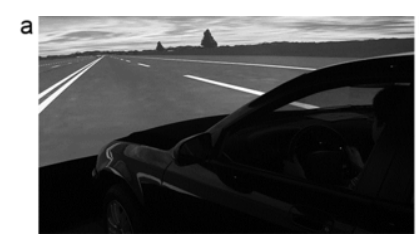

Fig. 1. Driving simulator: a.) Internal; b.) External.

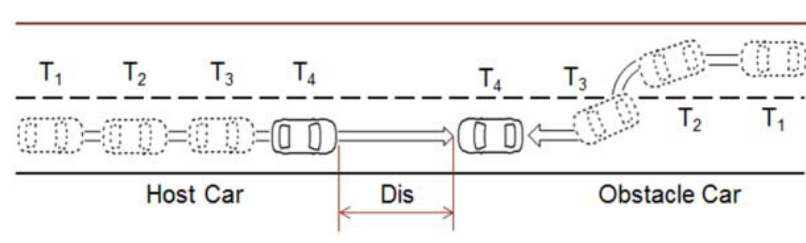

Fig. 2. Driving Scenario. 
consisted of many vehicles and barriers were placed on the edges of the road, preventing the driver from steering evasively when facing the collision. One obstacle car cut into the participant's lane at the moment the distance between the host car and the obstacle car reached a certain value. The obstacle car did not emerge until the first $10 \mathrm{~km}$, which was used to decrease the driver's awareness to the on-coming collision. The driving scenario is presented in Figure 2. At $T_{1}$ moment, the host and obstacle car started together. During $\mathrm{T}_{2}$ and $\mathrm{T}_{3}$ moments, the obstacle car cut into the host lane. At $\mathrm{T}_{4}$ moment, the obstacle car was fully in the host lane. The velocity of the obstacle car was set at 50 $\mathrm{km} / \mathrm{h}$.

The urgency level was characterized by the host car's speed $\left(\mathrm{V}_{0}\right)$ and the relative distance (Dis). The relative distance was defined as the distance between the host car and the obstacle car at the moment it was fully in the participant's lane. The $\mathrm{V}_{0}$ and Dis differed in various driving scenarios, and were considered as two independent factors. A higher $\mathrm{V}_{0}$ and smaller distance meant a greater urgency level. $\mathrm{V}_{0}$ was set at $25,50,75$, and $100 \mathrm{~km} / \mathrm{h}$ and Dis was set at 20,40,60, and 80 meters, which produced 16 collision scenarios. Each participant was required to drive in these 16 collision simulations in a random order. Twelve participants completed 192 driving scenarios in total. Prior to the experiment, each participant practiced in the driving simulator until he felt that he had adequate skills to control the vehicle. The participants were informed that only braking actions could be used to avoid collision. After completing four driving simulation tests, the participants rested for 15 minutes to avoid fatigue and drowsiness.

All drivers were informed about the procedure so they were aware of the on-coming emergencies [16]. They were young and no cell phones were allowed during driving, so fatigue and distractions were not factors in this study.

In emergency situations, the tibialis anterior muscle is considered the muscle that firstly reacts [17, 18], so EMGs of the drivers' tibialis anterior muscles were recorded and used to separate the premotor times and muscle activation times, which fractionated the braking reaction times more exactly.

\subsection{Braking reaction time}

The drivers' braking reaction times are shown in Figure 3. $\mathrm{T}_{\mathrm{OBS}}$ was defined as the moment when the obstacle car was fully in the host lane, shown as $\mathrm{T}_{4}$ in Figure $2 . \mathrm{T}_{\mathrm{EMG}}$ was defined as the moment when the EMG of the tibialis anterior muscle reached 5\% of its maximum value during the 16 drives for each participant, which indicated that the driver was preparing to move his foot to the brake pedal. $\mathrm{T}_{\mathrm{GOFF}}$ was defined as the moment when the accelerator aperture began to drop. If a collision occurred before $\mathrm{T}_{\mathrm{GOFF}}$, it indicated that the EMG signals were still conducting and it had not yet moved. $\mathrm{T}_{\mathrm{G} 0}$ was defined as the moment when the accelerator aperture reached zero. The lower extremity would be on the accelerator if the collision occurred before $\mathrm{T}_{\mathrm{G} 0}$. And it would be in the air if the collision occurred later than $\mathrm{T}_{\mathrm{G} 0}$. $\mathrm{T}_{\mathrm{BON}}$ was defined as the moment when the brake pedal force began to

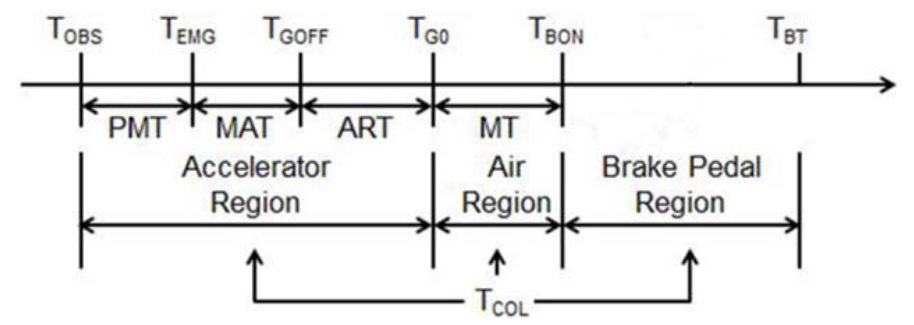

Fig. 3. Driver's braking reaction time history. 
increase, indicating the driver's foot on the brake pedal. $\mathrm{T}_{\mathrm{BT}}$ was defined as the moment when the host car stopped. $\mathrm{T}_{\mathrm{COL}}$ was defined as the time point when the two cars collided (Dis $=0$ ).

The drivers' braking reaction times were divided into pre-motor times (PMT), muscle activation times (MAT), accelerator release times (ART), and movement times (MT), as shown in Figure 3. As the collision might occur at any moment after the obstacle car fully cut in, the braking reaction time was divided into three regions when the collisions occurred: (1) Accelerator region, in which the lower extremity was located on the accelerator; (2) Air region, in which the lower extremity was in the air; (3) Brake pedal region, in which the lower extremity was located on the brake pedal.

\subsection{Data collection, statistics, and analysis}

The vehicle parameters were collected by the driving simulator, including $\mathrm{V}_{0}[\mathrm{~km} / \mathrm{h}], \mathrm{T}_{\mathrm{COL}}[\mathrm{ms}]$, and accelerator aperture [\%]. The driver's EMG at the tibialis anterior muscle of his right leg was recorded by a MP150 physiology recorder made by BioPac Systems, Inc (California, USA) and 40 $\mathrm{mm}$ diameter $\mathrm{Ag} / \mathrm{AgCl}$ disc electrodes (ConMed Co., Ltd, NY, USA). The electrodes were placed on the line $1 / 3$ of the way between the tip of the fibula and the tip of the medial malleolus. To reduce impedance at the electrode sites, the drivers' skin was shaved, wiped with 50/50 alcohol/distilled water, and coated with electrode gel. The sample frequency of the apparatus was $1000 \mathrm{~Hz}$. The EMG data were subjected to band pass of 10 to $350 \mathrm{~Hz}$. At the beginning of the test, a synchronous signal was sent to the EMG acquisition system by the driving simulator so that they recorded simultaneously.

The mean value and standard deviation were calculated for PMT, MAT, ART, and MT. Then they were subjected to two-way ANOVA and post-hoc pairwise comparisons using the tukey honest significant difference method. Pearson correlation analysis was performed between the braking reaction times and $\mathrm{V}_{0}$ and Dis. If a collision did not occur, the lower extremity was considered to be on the brake pedal. Binary logistical regression was used to predict the probabilities of the lower extremity being on the brake pedal and on the accelerator when a collision occurred. An alpha level of 0.05 was used for the statistical tests and Pearson correlation analysis.

\section{Results}

\subsection{Braking reaction times}

The fractionated braking reaction times are shown in Figure 4. It was found from two-way ANOVA that the main effect of $\mathrm{V}_{0}$ and the interaction effect of $\mathrm{V}_{0}$ and Dis had no significant effect on PMT (ANOVA, P > 0.05). However, the main effect of Dis to PMT was significant (ANOVA, $\mathrm{P}=$ 0.001). A significant difference on PMT was found from tukey post-hoc pairwise comparison between Dis of $80 \mathrm{~m}$ and $20 \mathrm{~m}$ (ANOVA, $\mathrm{P}=0.01$ ), and between $80 \mathrm{~m}$ and $40 \mathrm{~m}$ (ANOVA, $\mathrm{P}=$ 0.001). Main effects and interaction effects of $\mathrm{V}_{0}$ and Dis were not significant on MAT, ART, or MT (ANOVA, $\mathrm{P}>0.05$ ).

The results of the correlation analyses are shown in Table 1 . MT (ANOVA, $P=0.01$ ) was negatively related to $\mathrm{V}_{0}$ and the correlation was significant. PMT (ANOVA, $\mathrm{P}<0.001$ ) and MAT (ANOVA, $\mathrm{P}=0.001$ ) showed positive relations to Dis and the correlations were significant. RT+MAT (ANOVA, $\mathrm{P}>0.05 ; \mathrm{P}<0.001$ ), ART+MT (ANOVA, $\mathrm{P}<0.05 ; \mathrm{P}>0.05$ ) and $\mathrm{RT}+\mathrm{MAT}+\mathrm{ART}+\mathrm{MT}$ (ANOVA, $\mathrm{P}<0.05 ; \mathrm{P}<0.001$ ) showed negative correlations to $\mathrm{V}_{0}$ and showed positive correlations to Dis. 

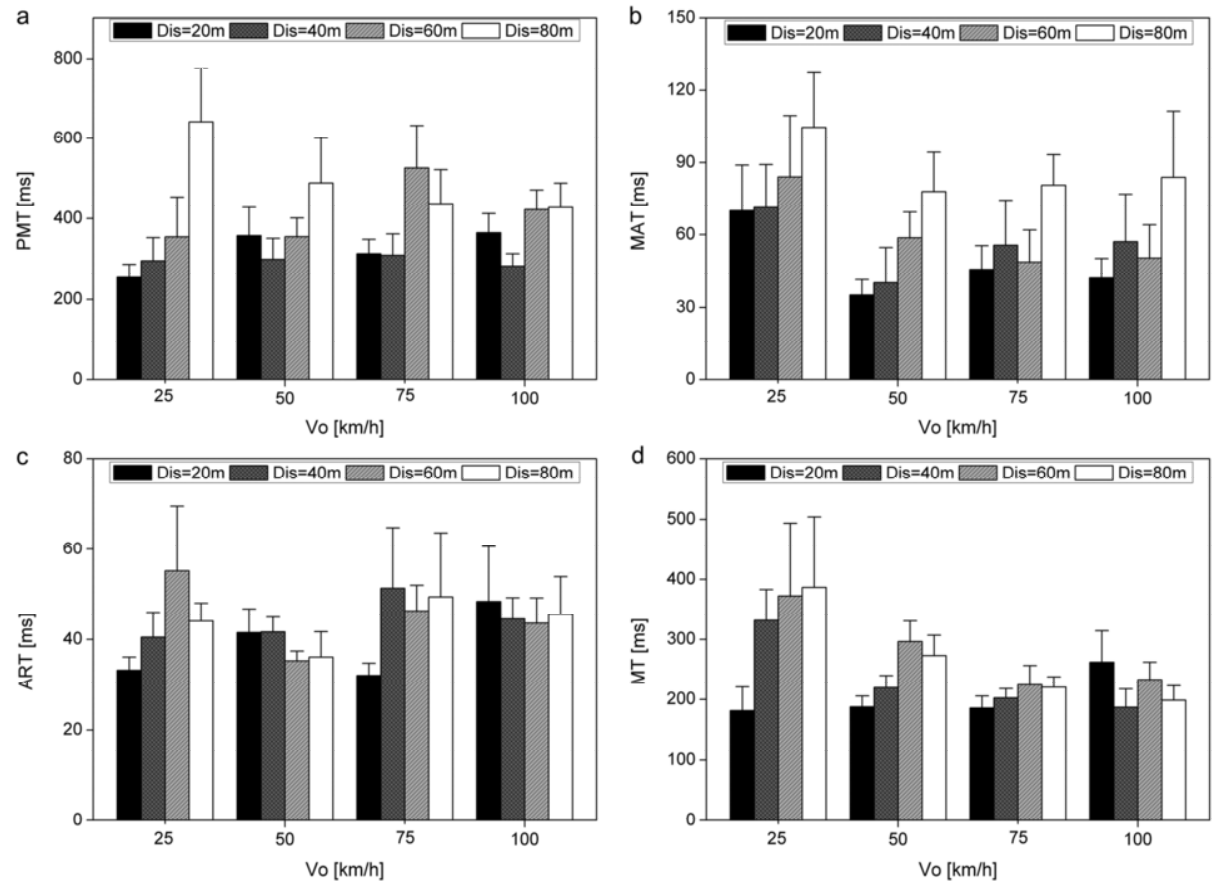

Fig. 4. Braking reaction times: Bars and lines show means and standard error of the mean (SEM).

Table 1

Pearson correlation analysis

\begin{tabular}{llllllll}
\hline $\mathrm{r}$ & PMT & MAT & ART & MT & PMT+MAT & ART+MT & PMT+MAT+ART+MT \\
\hline $\mathrm{V}_{0}$ & -0.040 & -0.127 & 0.052 & $-0.281^{*}$ & -0.064 & $-0.259^{*}$ & $-0.191^{*}$ \\
Dis & $0.326^{*}$ & $0.281^{*}$ & 0.085 & 0.169 & $0.368^{*}$ & 0.172 & $0.381^{*}$ \\
\hline \multicolumn{1}{c}{ Note: ${ }^{*}$ Correlation is significant at the 0.05 level (2-tailed). }
\end{tabular}

\subsection{Breaking behavior of lower extremity}

The braking behaviors of the drivers' lower extremities when facing emergencies were classified based on the fraction of braking reaction times and the comparison between collision times and braking reaction times. When facing on-coming collisions at different urgency levels, there were two spatial relations between the two cars: one was that the host car stopped with a probability of $36.5 \%$ (70 of 192 drives), and the other was collision (63.5\%, 122 of 192 drives). The reaction characteristics and braking performances (pre-crash posture) of the drivers' lower extremities varied with changes of urgency levels. The collisions occurred before total braking or even before the start of braking. At the collision moment, the probability of the driver's lower extremity being on the accelerator was $7.4 \%$ ( 9 of 122 drives), being in the air was $18.9 \%$ (23 of 122 drives), and being on brake pedal was $73.7 \%$ (90 of 122 drives). In other words, a driver might collide in different lower extremity postures. The drivers' reaction characteristics in the four typical conditions mentioned above are shown in Figure 5, where $\mathrm{T}_{\mathrm{OBS}}$ denoted zero time point. The relationship between time and collision trigger signal, $\mathrm{EMG}$, host car speed, accelerator aperture, and brake pedal force are shown. The collision trigger signal changed from high $(5 \mathrm{~V})$ to low $(0 \mathrm{~V})$ when a collision occurred. 

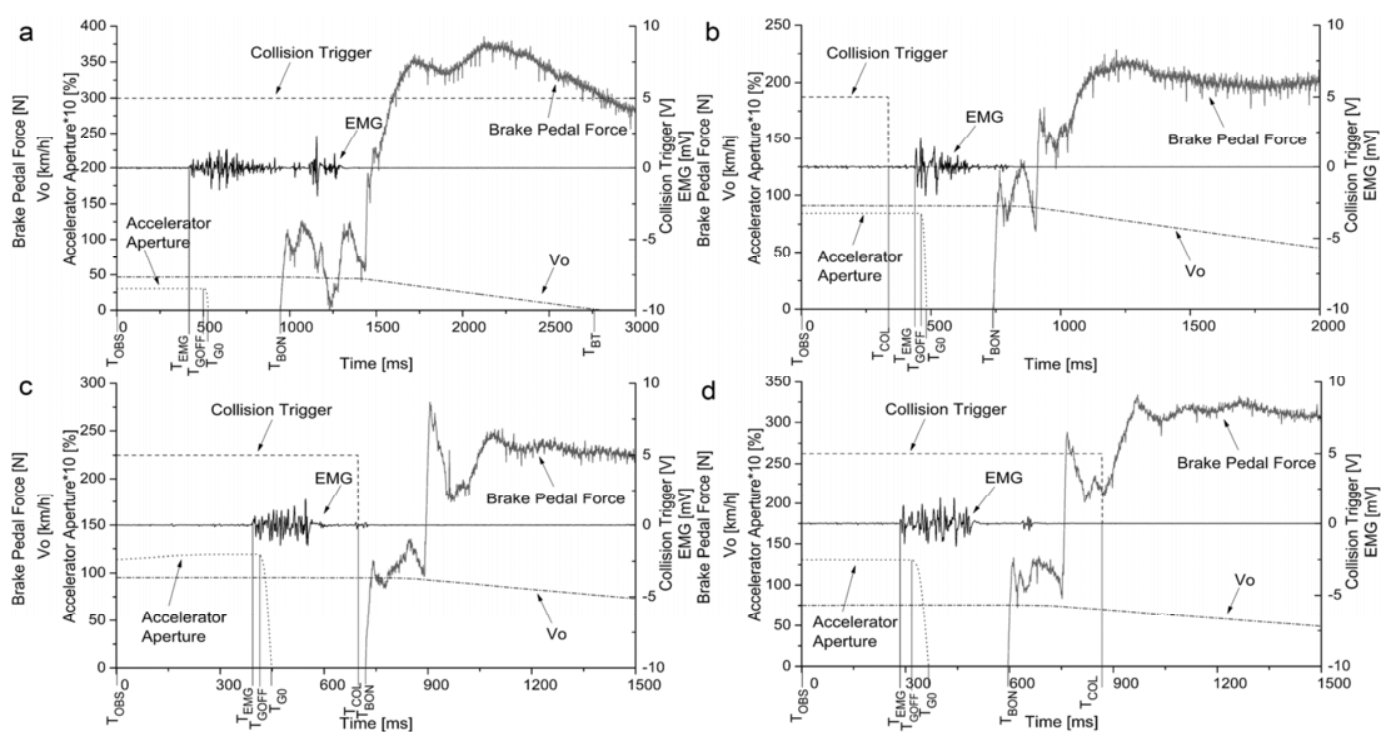

Fig. 5. Driver's reaction characteristics: a.) Total braking: The collision trigger signal was in high level (5V) from beginning to end, indicating the host car stopped before collision; b.) Accelerator region: collision trigger signal changed before the lower extremity totally released the accelerator $\left(\mathrm{T}_{\mathrm{COL}}<\mathrm{T}_{\mathrm{G} 0}\right)$, which indicated that it was in the accelerator region when collision occurred; c.) Air region: the collision trigger signal changed before the lower extremity depressed the brake pedal $\left(\mathrm{T}_{\mathrm{G} 0}<\mathrm{T}_{\mathrm{COL}}<\mathrm{T}_{\mathrm{BON}}\right)$, which indicated that it was in the air region when collision occurred; d.) Brake pedal region: collision trigger signal changed after the lower extremity depressed the brake pedal $\left(\mathrm{T}_{\mathrm{COL}}>\mathrm{T}_{\mathrm{BON}}\right)$, which indicated that it was in the brake pedal region when collision occurred. The value of accelerator aperture was 10 times amplified for better illustration.

The drivers' braking reaction times in the nine drives that resulted in collisions while in the accelerator region are shown in Table 2. Among them, four (participants 4, 5, 6, and 9) occurred before the muscle of the driver's lower extremity activated, three (participants 1,8 , and 11) occurred between the onset of the lower extremity muscle activation and the beginning of the release of the accelerator, and two (participants 7 and 12) occurred when the lower extremity was releasing the accelerator.

The results of binary logistical regression are shown in Figure 6. A significant influence of Wald test for $\mathrm{V}_{0}$ and Dis was found for the probabilities of collision and the driver's lower extremity on the brake pedal $(\mathrm{P}<0.001)$. The effect of $\mathrm{V}_{0}$ on the probability of the driver's lower extremity on the accelerator $(\mathrm{P}<0.01)$ was significant, and Dis had little influence on it $(\mathrm{P}>0.05)$.

Table 2

Drivers' braking reaction times

\begin{tabular}{lllllll}
\hline No. & $\mathrm{V}_{0}[\mathrm{~km} / \mathrm{h}]$ & Dis $[\mathrm{m}]$ & $\mathrm{T}_{\mathrm{EMG}}[\mathrm{ms}]$ & $\mathrm{T}_{\mathrm{GOFF}}[\mathrm{ms}]$ & $\mathrm{T}_{\mathrm{G} 0}[\mathrm{~ms}]$ & $\mathrm{T}_{\mathrm{COL}}[\mathrm{ms}]$ \\
\hline 1 & 100 & 20 & 333 & 344 & 382 & 335 \\
4 & 100 & 20 & 472 & 502 & 529 & 294 \\
5 & 100 & 20 & 402 & 451 & 483 & 335 \\
6 & 100 & 20 & 499 & 570 & 609 & 332 \\
7 & 100 & 20 & 253 & 278 & 410 & 318 \\
8 & 75 & 20 & 352 & 442 & 472 & 415 \\
9 & 100 & 20 & 456 & 505 & 540 & 322 \\
11 & 100 & 20 & 345 & 398 & 423 & 371 \\
12 & 75 & 20 & 233 & 259 & 398 & 329 \\
\hline
\end{tabular}

Note: The driver's lower extremity was in the accelerator region. 

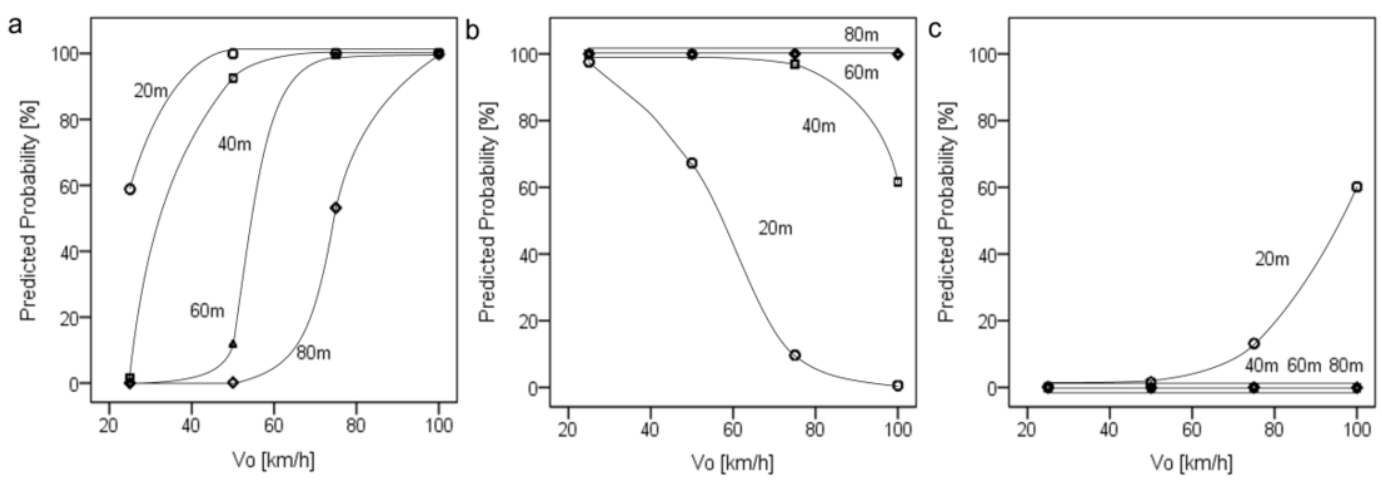

Fig. 6. Binary logistical regression: a.) Collision probability; b.) Probability of lower extremity on brake pedal at the collision moment; c.) Probability of lower extremity on accelerator at the collision moment.

The faster the vehicle speed and the shorter the relative distance are, the larger the probability of collision and the lower extremity being on the accelerator are. A slower speed and a longer distance led to a larger probability of the lower extremity being on the brake pedal. In other words, the urgency level related to $\mathrm{V}_{0}$ and Dis affected the posture of the driver's lower extremity. It changed with the increase of collision urgency level, which was from the depression of the brake pedal to no support in the air, and then the depression of the accelerator. The faster the speed and the shorter the distance are, the larger the probability of the lower extremity being in the air or even on the accelerator.

\section{Discussion}

A driver's response consists of braking reaction time and braking behavior. The former represents the reaction ability and the latter is the pre-crash posture of a driver's lower extremity, which affects the musculoskeletal characteristics when facing an on-coming collision. A better understanding of kinematic behaviors and their occurrence times will provide a reference for intervention times of vehicle safety systems and injury biomechanics analysis $[3,6]$.

Braking reaction times are affected by many factors, such as age [19], gender [20], awareness [16], and urgency level [11]. In order to investigate the influence of urgency level (vehicle speed and relative distance) on braking reaction times, other factors were limited in this study. The drivers' braking reaction times were fractionated by introducing EMG data from the drivers' tibialis anterior muscles.

Many previous studies divided a driver's braking reaction time into three parts including the brake reaction time (from the onset of the brake lights of the obstacle car to the release of the accelerator of the host car), movement time (from the release of the accelerator to the depression of the brake pedal of the host car), and total brake time (the sum of the former two). In this study, the mean time from the obstacle car being fully in the host car's lane to the lower extremity of the driver starting to release the accelerator (PMT+MAT) was $393 \mathrm{~ms}$ when $\mathrm{V}_{0}=50 \mathrm{~km} / \mathrm{h}$ and Dis $=20 \mathrm{~m}$, which was comparable to $376 \mathrm{~ms}$ when $\mathrm{V}_{0}=50 \mathrm{~km} / \mathrm{h}$ and Dis $=23 \mathrm{~m}$ obtained in a similar condition [19]. The total brake time (PMT+MAT+ART+MT, $622 \mathrm{~ms}$ ) was comparable to former research results (569 ms in Martin's research [19], and $633 \mathrm{~ms}$ in Olson's [7]). Regarding risk time (0.36 to $5.76 \mathrm{~s}$ in this study), the mean of PMT+MAT was from 350 to $750 \mathrm{~ms}$, the mean of ART+MT was from 210 to $450 \mathrm{~ms}$ and the mean of PMT+MAT+ART+MT was from 590 to $1200 \mathrm{~ms}$, which was also comparable to other studies [6, 
$11]$.

The onset time when a driver applied the brake, i.e., the moment when the muscle activated, was detected by EMG. Combining this with the method based on vehicle parameters, the drivers' breaking responses to collisions were obtained 35 to $105 \mathrm{~ms}$ ahead, as shown in Figure 4(b). This coincided with former studies $[17,18]$.

The drivers' reaction times were comparable to previous studies when it was fractionated using the traditional method $(\operatorname{tra}($ or $\operatorname{trg})=\mathrm{PMT}+\mathrm{MAT}, \operatorname{trm}=\mathrm{ART}+\mathrm{MT}, \operatorname{trb}($ or trh $)=\mathrm{PMT}+\mathrm{MAT}+\mathrm{ART}+\mathrm{MT})$ $[6,11]$. After introducing the EMG, the drivers' reaction times were divided into PMT, MAT, ART, and MT. Their influences on different driving speeds and relative distances were noted. PMT, MAT, and MT decreased with the increase of vehicle speed. ART was regarded as a constant with vehicle speed change. All divisions of braking reaction times increased with the relative distance. This indicated that every division of the reaction times should be considered in the design of vehicle active safety systems under different emergency situations. This also implied that the reaction times could be recorded efficiently in a driving simulator under different driving situations, and the introduction of EMG did not have a significant effect on the trend of braking reaction times.

When facing on-coming collisions at different urgency levels, a driver can be in various braking behaviors and musculoskeletal characteristics such as bracing or muscle tensing. The change of a driver's posture can have a great influence on the driver's lower extremity position and muscle activation status, and can also affect injuries [3, 4, 14]. During a collision, the lower extremity position is different based on braking strategies. A driver's foot suffers various loads and behaviors depending on where it is located on the brake pedal (center or edge) [21]. Additionally, the change of muscle force affects the injuries of the ankle joint and Achilles tendon, and the muscle activation level can exacerbate axial loading injuries [14, 15]. However, it is still unclear what posture the lower extremity is in when an on-coming collision occurs.

A driver's lower extremity is commonly thought to be on the brake pedal in most collision injury investigations $[3,5]$. However, this is not always true in actual situations, as the driver cannot always react in driving situations that exceed the physiological limit of human reaction capabilities [22, 23]. The common braking motion is to release the accelerator and then push the brake, a period that lasts between 420 and $730 \mathrm{~ms}$ [12]. However, a collision can occur at any time, and the release of the accelerator doesn't begin until at least $250 \mathrm{~ms}$ after the emergency is recognized [24]. It takes at least $80 \mathrm{~ms}$ for the change from rest state to full activation state of the muscle [25]. As the foot does not move until it is fully activated, there is a $170 \mathrm{~ms}$ time span for the crash to occur in the inactivated stage with the foot on the accelerator. Moreover, the muscle activation status of the lower extremity varies in driving situations with different emergency levels. Therefore, it is important to measure the very moment of muscle activation during the braking motion, especially the accelerator response, at different values of speed and distance parameters.

Thus, EMGs of the tibialis anterior muscles were introduced and the activation times were measured in this study. The moment that the EMG rose sharply was one of the references points when analyzing the braking behavior of the lower extremity, which was used to determine the muscle activation status when the crash occurred. The braking reaction times were divided into three regions: accelerator, air, and brake pedal. The collisions could occur in any region, particularly in the accelerator region at any one of the three muscle activation stages: inactivated, being activated, and fully activated.

In some conditions, the muscle of the lower extremity was not be fully activated in the accelerator region, as was seen in participants 1,8 , and 11 in Table 2, $\mathrm{T}_{\mathrm{EMG}}<\mathrm{T}_{\mathrm{COL}}<\mathrm{T}_{\mathrm{GOFF}}$. Although the muscle was activated, it was not strong enough to move the lower extremity. The foot was still on the 
accelerator while the muscle was being activated. Even if the driver was reacting to the emergency, the foot was possibly still on the accelerator (participants 7 and $12, \mathrm{~T}_{\mathrm{GOFF}}<\mathrm{T}_{\mathrm{COL}}<\mathrm{T}_{\mathrm{G} 0}$ ), according to the pedal travel. Moreover, if the situation is extremely urgent, such as with $100 \mathrm{~km} / \mathrm{h}$ speed and $20 \mathrm{~m}$ relative distance, some drivers (participants 4, 5, 6, and 9) were unable to react in time (the collision happened before they reacted). In these cases, the driver had not changed to a pre-crash posture and remained in a normal driving posture when the crash occurred. Therefore, it was necessary to take the driver's musculoskeletal characteristics, such as braking behavior and muscle activation status, into account for further sport mechanics and collision injury analysis.

Several limitations existed for the results of this study. Firstly, the effects of age and gender were not considered. Drivers' responses for female and elderly participants will be investigated and their differences between young men will be compared in the future. Secondly, participants did not suffer from any cognitive workload such as radio or telephone. These will be included in a following research project.

\section{Conclusions}

The drivers' braking reaction times and braking behaviors were studied while facing front-coming vehicle collisions at different urgency levels, i.e., at different vehicle speeds and relative distances. A driving simulator was used as a testing platform to simulate traffic collision scenarios and to record the drivers' kinematic behaviors. Twelve male drivers were recruited for the braking reaction tests. Based on the vehicle parameters and the EMGs of the drivers' lower extremities, their braking reaction times were fractionated into pre-motor time, muscle activation time, accelerator release time, and movement time. The influence of speed and relative distance was analyzed on every fraction of the braking reaction times. The braking behaviors involving the lower extremities while facing different collision scenarios were studied through comparisons between the collision times and braking reaction times. It was found that:

(1) Combining the measuring methods based on the vehicle parameters and the EMGs of the drivers' lower extremities, their breaking responses to vehicle collisions were obtained 35 to $105 \mathrm{~ms}$ ahead.

(2) Movement times $(\mathrm{r}=-0.281, \mathrm{P}=0.01)$ were negatively related to vehicle speed. The PMTs $(\mathrm{r}=0.326, \mathrm{P}<0.001)$ and MATs $(\mathrm{r}=0.281, \mathrm{P}=0.001)$ were positively related to relative distance.

(3) The possibility of the driver's lower extremity being on the accelerator was $7.4 \%$, in the air was $18.9 \%$, or on the brake pedal was $73.7 \%$ at the moment of collision.

(4) When a collision occurred at a higher urgency level, the driver's lower extremity was more likely to be in the air or on the accelerator. If the collision occurred at a higher vehicle speed and smaller relative distance, the driver will be unable to react and keep in a normal driving posture which muscles are not or not fully activated.

\section{Acknowledgments}

This study was supported by 973 Program under Grant No. 2012CB723802; Specialized Research Fund for the Doctoral Program of Higher Education under Grant No. 20120061110028; Jilin Provincial Research Foundation for Technology Guidance under Grant No. 20130413058GH and Grant No. 20150204055GX and ASCL Grant under Grant No. 20120104. 


\section{References}

[1] D.V. McGehee and O.M.J. Carsten, Perception and biodynamics in unalerted precrash response, Proceedings of 54th Annual Scientific Conference of the Association for the Advancement of Automotive Medicine 54 (2010), 315-331.

[2] S. Ejima, Y. Zama, K. Ono, K. Kaneoka, I. Shiina and H. Asada, Prediction of pre-impact occupant kinematic behavior based on the muscle activity during frontal collision, Proceedings of the 21 st International Technical Conference on the Enhanced Safety of Vehicles, Stuttgart, Germany, 2009, pp. 1-11.

[3] A. Hault-Dubrulle, F. Robache, M.P. Pacaux and H. Morvan, Determination of pre-impact occupant postures and analysis of consequences on injury outcome, Part I: A driving simulator study, Accident Analysis \& Prevention 43 (2011), 66-74.

[4] M. Behr, G. Poumarat, T. Serre, P.J. Arnoux, L. Thollon and C. Brunet, Posture and muscular behaviour in emergency braking: An experimental approach, Accident Analysis \& Prevention 42 (2010), 797-801.

[5] D. Bose, J.R. Crandall, C.D. Untaroiu and E.H. Maslen, Influence of pre-collision occupant parameters on injury outcome in a frontal collision, Accident Analysis \& Prevention 42 (2010), 1398-1407.

[6] R.S. Jurecki, M. Jaśkiewicz, M. Guzek, Z. Lozia and P. Zdanowicz, Driver's reaction time under emergency braking: A car-research in a driving simulator, Eksploat Niezawodn 14 (2012), 295-301.

[7] P.L. Olson and M. Sivak, Perception-response time to unexpected roadway hazards, Human Factors 28 (1986), 91-96.

[8] A.D. Weiss, The locus of reaction time change with set, motivation, and age, Journal of Gerontology 20 (1965), 60-64.

[9] A. Mero and P.V. Komi, Reaction time and electromyographic activity during a sprint start, European Journal of Applied Physiology 61 (1990), 73-80.

[10] H.Y. Choi, S.J. Sah, B. Lee, H.S. Cho, S.J. Kang, M.S. Mun, I. Lee and J. Lee, Experimental and numerical studies of muscular activations of bracing occupant, Proceedings of the 19th International Technical Conference on the Enhanced Safety of Vehicles, Washington, D.C., USA, 2005, pp. 1-10.

[11] R.S. Jurecki, T.L. Stańczyk and M.J. Jaśkiewicz, Driver's reaction time in a simulated, complex road incident, Transport (2014), 1-11.

[12] M. Green, "How long does it take to stop?" Methodological analysis of driver perception-brake times, Transportation Human Factors 2 (2000), 195-216.

[13] J.R. Brault, G.P. Siegmund and J.B. Wheeler, Cervical muscle response during whiplash: Evidence of a lengthening muscle contraction, Clinical Biomechanics 15 (2000), 426-435.

[14] E.C. Hardin, A. Su and A.J. van den Bogert, Foot and ankle forces during an automobile collision: The influence of muscles, Journal of Biomechanics 37 (2004), 637-644.

[15] E.C. Hardin, A. Su and A.J. van den Bogert, Pre-impact lower extremity posture and brake pedal force predict foot and ankle forces during an automobile collision, Journal of Biomechanical Engineering 126 (2004), 770-778.

[16] N. Schweitzer, Y. Apter, G. Ben-David, D.G. Liebermann and A. Parush, A field study on braking responses during driving, II. minimum driver braking times, Ergonomics 38 (1995), 1903-1910.

[17] Y. Seto, K. Minegishi, Z. Yang and T. Kobayashi, Research on detection of braking reactions in emergency situations, Vehicle System Dynamics Supplement 41 (2004), 784-790.

[18] P.M. D'Addario, B. Donmez and K.W. Ising, EMG provides an earlier glimpse into the effects of cognitive distraction on brake motor response, Proceedings of the Human Factors and Ergonomics Society Annual Meeting 58 (2014), 2200-2204.

[19] P.L. Martin, T. Audet, H. Corriveau, M. Hamel, M. D'Amours and C. Smeesters, Comparison between younger and older drivers of the effect of obstacle direction on the minimum obstacle distance to brake and avoid a motor vehicle accident, Accident Analysis \& Prevention 42 (2010), 1144-1150.

[20] L. Warshawsky-Livne and D. Shinar, Effects of uncertainty, transmission type, driver age and gender on brake reaction and movement time, Journal of Safety Research 33 (2002), 117-128.

[21] R.W. Rudd, J.R. Crandall, C.R. Bass, S. Lynn and J. Keller, Lower extremity and brake pedal interaction in frontal collisions: Sled tests, SAE Technical Paper, 1998, 980359.

[22] BD. Stemper, N. Yoganandan, R.D. Rao and F.A. Pintar, Reflex muscle contraction in the unaware occupant in whiplash injury, Spine 30 (2005), 2794-2798.

[23] J.T. Viitasalo and P.V. Komi, Interrelationships between electromyographic, mechanical, muscle structure and reflex time measurements in man, Acta Physiologica Scandinavica 111 (1981), 97-103.

[24] G.R., Wright and R.J. Shephard, Brake reaction time-effects of age, sex, and carbon monoxide, Archives of Environmental Health: An International Journal 33 (1978), 141-150.

[25] B.D. Stemper, N. Yoganandan, J.F. Cusick and F.A. Pintar, Stabilizing effect of precontracted neck musculature in whiplash, Spine 31 (2006), E733-E738. 